\title{
Entretien
}

\section{Michel Badré : « la forêt au rythme des sciences et de la société »}

\section{Propos recueillis par Henri Décamps}

\author{
Michel Badréa ${ }^{a}$ Henri Décamps ${ }^{b}$ \\ a Inspection générale de l'environnement, Ministère de l’Écologie et du Développement durable, 20 avenue de Ségur, \\ 75302 Paris 07 SP, France \\ b Écologue, CNRS, Laboratoire Dynamique de la biodiversité, CNRS, 29 rue Jeanne Marvig, 31055 Toulouse cedex 04, France
}

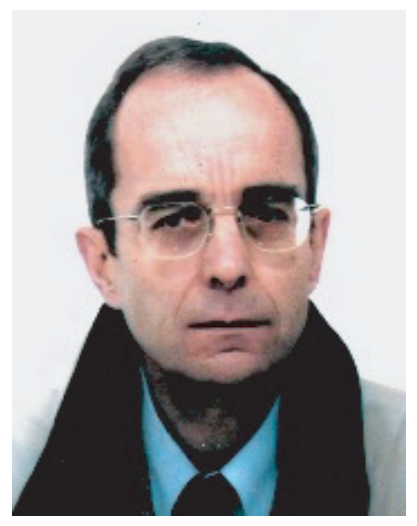

Éléments de biographie

Né en 1948, ingénieur du Génie rural, des eaux et des forêts, Michel Badré est entré à l'Office national des forêts en 1972 et y a effectué presque toute sa carrière, dans différents postes de terrain puis comme directeur régional en Champagne-Ardenne et en Franche-Comté, et enfin comme directeur général adjoint de 1999 à 2002, après les tempêtes et pendant la grande réorganisation de l'établissement qui a suivi. Il a également dirigé l'Institut pour le développement forestier, et le groupement d'intérêt public «Écosystèmes forestiers » (GIP Écofor) en 2003 et 2004. Il est membre de l'Inspection générale de l'environnement depuis 2002.
Henri Décamps (NSS) : En juillet 2002, dans un article paru dans Le Monde, vous écriviez : "Les forestiers ne peuvent dire seuls ce qui est bon pour la forêt, et la forêt n'a pas vraiment besoin de forestiers pour vivre : elle a prouvé le contraire pendant des millions d'années. C'est la société qui a besoin des forestiers, et qui seule leur donne une légitimité, en leur fixant des objectifs clairs, pour répondre à ses attentes. » Ceci nous donne un point de départ idéal pour un entretien dans NSS : de quel recul dispose-t-on pour comprendre le rôle des forestiers dans la société?

Michel Badré : Ce recul est faible : on ne parle réellement de technique sylvicole en Europe que depuis 200 ans, à peine plus, même pas deux générations de chêne... Le taillis, beaucoup plus ancien, n'était

Auteur correspondant:

M. Badré, michel.badre@ecologie.gouv.fr qu'une organisation des prélèvements assimilables à de la cueillette, pour limiter les pénuries. Et le schéma évoqué dans Le Monde était plus une vision idéale du pilotage d'un service collectif par le corps social, que la description d'une règle d'usage. Rares sont en effet les cas où la société a, par une voix autorisée, dit explicitement aux forestiers ce qu'elle voulait.

À cet égard, les forestiers citent souvent l'exemple de Colbert. Après la grande enquête qu'il fait mener sur l'état des forêts, Colbert exprime en effet avec force, dans son ordonnance de 1669, l'exigence de revenir à une gestion durable ${ }^{1}$ de la ressource en bois de chauffage, et de développer des techniques permettant de répondre à des besoins stratégiques en matière de bois de marine. La réponse, en termes de technique forestière, ne viendra

\footnotetext{
1 Au sens actuel : répondre aux besoins d'aujourd'hui, sans compromettre la capacité à répondre à ceux de demain. . .
} 
réellement qu'un siècle plus tard, avec Duhamel du Monceau (1700-1782), fondateur d'une science forestière nouvelle, tournée vers la maîtrise qualitative de la production, à partir d'observations naturalistes.

$C^{\prime}$ est une révolution : le métier de forestier s'était constitué jusque-là en Europe de 1'Ouest comme un métier de police et de contrôle, avec ses procédures et ses tribunaux spéciaux, pour maîtriser une demande de boisénergie (pour le chauffage et l'industrie : forges, verreries, etc.) et de droits d'usage en forêt, par des pratiques d'assolement assez rudimentaires - une demande croissante avec la diminution des surfaces forestières, sous l'effet des défrichements agricoles.

Au XIX ${ }^{\mathrm{e}}$ siècle, l'école forestière allemande, et Lorentz et Parade à Nancy, poursuivront l'élaboration de la sylviculture, fondée par Duhamel du Monceau quelques décennies plus tôt.

H.D. : Peut-on évoquer d'autres exemples plus récents?

Michel Badré : J'en vois deux. D'abord, dans le domaine des services, l'exemple de la restauration des terrains en montagne (RTM), politique mise en place à la fin du XIXe siècle pour enrayer les dommages dus au surpâturage en montagne. Alertée par quelques techniciens (notamment Surell, un ingénieur des Ponts et Chaussées soucieux de sécuriser les voies de communication contre les crues torrentielles), la puissance publique charge les forestiers d'appliquer une loi votée en 1860 et qui privilégie clairement l'intérêt général sur les intérêts des éleveurs locaux. Les vifs conflits qui en résultent inciteront le gouvernement et le Parlement à tempérer cette politique, sans la remettre en cause, par une nouvelle loi de 1882. Mais les tensions entre le corps forestier et les populations de montagne ${ }^{2}$ ne s'apaiseront réellement que faute de combattants, quand l'exode rural, d'une part, et la perte de moyens de l'administration forestière, $d^{\prime}$ 'autre part, auront réduit un par un tous les conflits locaux, entre les deux guerres mondiales.

L'autre exemple est celui de la politique de reboisements définie après 1945 avec la création du Fonds forestier national (FFN). Ici encore, les forestiers assurent la mise en œuvre d'une politique volontariste dans un cadre législatif et politique explicite. Il s'agissait d'abord de garantir un approvisionnement autonome en papier journal (majoritairement contrôlé dans les années 1950 par les États-Unis et le Canada d'une part, le bloc soviétique de l'autre - ce que des gouvernants de «troisième

\footnotetext{
2 Tensions est un mot faible : sur ces conflits liés à la RTM, voir notamment : Kalaora, B., Savoye, A., 1986. La Forêt pacifiée, sylviculture et sociologie au XIX ${ }^{e}$ siècle, Paris, L'Harmattan; Ollagnon, H., 1982. La RTM dans une France décentralisée : plusieurs acteurs pour un dessein commun, Revue forestière française, XXXIV, $5,213-238$; H. Ollagnon réalisa en outre, au sein des services RTM, un audit patrimonial pour le compte du ministère de l'Agriculture.
}

voie » ne pouvaient accepter). Il s'agissait ensuite, à plus long terme, de répondre à un autre besoin stratégique : soutenir l'effort de construction. Bras séculier de cette politique publique, les forestiers en subiront le contrecoup dans les années 1970 : les plantations devenues alors visibles dans le paysage susciteront des réactions parfois vives, alors même que la pertinence stratégique des objectifs de base était devenue moins évidente.

Dans le cas de Colbert comme dans ceux de la RTM et du FFN, la demande sociale s'exprime par une politique publique, reposant sur des décisions gouvernementales ou parlementaires, que les forestiers sont chargés d'appliquer. Cette «demande sociale » exprimée au corps forestier par l'État, et formulée par lui dans son rôle de représentant de l'intérêt général, peut d'ailleurs être très significativement différente de celle exprimée directement par la société dans son expression de base : l'exemple de la RTM est significatif à cet égard.

H.D. : La demande sociale peut s'exprimer de la même façon en d'autres milieux, celui des eaux continentales par exemple. N'est-ce pas encore le cas général?

Michel Badré : Non car, surtout plus récemment, la société s'adresse aux forestiers de façon beaucoup moins structurée, souvent par des inquiétudes diffuses qui s'amplifient jusqu'à devenir assourdissantes, même si elles ne sont reprises dans aucune orientation politique explicite. Le premier exemple, déjà cité, est celui de l'hostilité aux plantations résineuses, dans les années 1960-1970. Le deuxième, plus significatif (on y reviendra) est celui des pluies acides, au début des années 1980 : les craintes de la société, relayées par une sensibilité politique plus forte dans le monde germanique, seront traduites dans des décisions politiques européennes avant d'être reprises en France. Le même schéma se reproduit de façon très emblématique aux États-Unis dans les années 1980-1990, avec le conflit de la chouette tachetée. Le choix privilégiant la protection de cette chouette dans les massifs forestiers de l'Ouest des États-Unis, en opposition aux intérêts des industries du bois, donnera lieu à un très vif débat, finalement tranché en faveur de la chouette par le président des États-Unis lui-même ${ }^{3}$.

Les forestiers étaient habitués à un mode de fonctionnement hérité en droite ligne de la période où l'État technicien était le maître d'œuvre de sa propre politique : en gros, du XIX ${ }^{\mathrm{e}}$ siècle aux années 1960. Dans ce schéma, probablement mort avec la fin des trente glorieuses, l'administration technique met en œuvre les politiques explicites de l'État : construire un réseau ferré, un armement nucléaire, le Concorde ou, plus modestement, mettre en œuvre la politique RTM ou développer les boisements résineux productifs. Lorsqu'il n'y a pas de politique explicite, l'administration technique les définit elle-même

\footnotetext{
3 À l'époque, Bill Clinton et pas son prédécesseur ni son successeur, dont nul ne sait quels arbitrages ils auraient rendus.
} 
(ce qui est l'essence même de la technocratie), quitte à se défendre avec énergie lorsqu'elle est ensuite attaquée par le public. Mais les forestiers ont été beaucoup plus mal à l'aise face aux situations où la demande sociale s'exprime par des voix jugées, en première analyse, autoproclamées ou illégitimes, alors que les pouvoirs publics vers lesquels on se tourne alors ne disent rien... C'est cette difficulté, très centrée sur les modes de construction et de conduite collective du débat public qu'il faut maintenant savoir résoudre.

H.D. : La gestion forestière a donc fortement évolué depuis ses débuts jusqu'à nos jours. Pouvez-vous préciser quelles étapes marquent cette évolution?

Michel Badré : Je répondrai ici, comme aux autres questions, en forestier français, et avec mon expérience personnelle qui est surtout celle d'un forestier de l'ONF, gestionnaire de forêts publiques. Au commencement, pour le bois comme pour les produits alimentaires, règne la cueillette - prélèvement de bois sans souci de reconstitution. Vers le Moyen Âge, la raréfaction des surfaces productives et l'augmentation des besoins conduisent à réguler les prélèvements en inventant, pour les essences feuillues, l'exploitation en taillis : une rotation géographique et chronologique des parcelles permet alors aux rejets de souche de reconstituer un stock ligneux suffisant entre deux passages en coupe. Vers le XIV et le $\mathrm{XV}^{\mathrm{e}}$ siècle, les mêmes besoins croissants de bois pour l'énergie conduisent à un début de formalisation de la culture des résineux, dans le Nord-Est, ou du chêne, dans l'Ouest de la France.

Mais ce n'est qu'aux XVIII ${ }^{e}$ et XIX ${ }^{e}$ siècles - avec Duhamel du Monceau, puis Lorentz et Parade et l'école forestière de Nancy, comme on l'a vu - que naît vraiment la sylviculture, technique de gestion des peuplements forestiers orientée vers certains types de production. D'inspiration exclusivement naturaliste au début, cette technique se complète au XIX ${ }^{\mathrm{e}}$ siècle avec l'apparition et le perfectionnement des méthodes d'aménagement : à la prise en compte de la physiologie de l'arbre, on ajoute la volonté d'inscrire dans le temps et dans l'espace la dynamique du peuplement forestier, à des échelles de plus en plus larges ${ }^{4}$.

Dans la deuxième moitié du XIX ${ }^{\mathrm{e}}$ siècle, l'écologie scientifique trouve très vite son application dans la foresterie, sous une forme très finalisée vers la production. Dans la droite ligne des préceptes des pères fondateurs de la sylviculture («imiter la nature, hâter son œuvre»), on cherche comment utiliser au mieux les caractéristiques

\footnotetext{
${ }^{4}$ Au-delà du développement des aménagements des forêts domaniales, à partir du milieu du XIX ${ }^{\mathrm{e}}$ siècle, on signalera ici la première tentative de description quantitative de l'ensemble des massifs forestiers français, avec l'inventaire Daubrée publié en 1912, une quarantaine d'années avant la systématisation de cette démarche par la création de l'Inventaire forestier national (IFN).
}

des écosystèmes pour les mettre au service des objectifs de production. Le développement de la pédologie forestière au $X X^{\mathrm{e}}$ siècle, avec Duchaufour, ou celui des typologies de stations à partir des années 1970 sont probablement les exemples les plus aboutis de cette utilisation approfondie de l'écologie au service des techniques forestières, pour optimiser la qualité et la quantité des bois produits. D'ailleurs, cette utilisation des connaissances écologiques pour tenter d'optimiser les fonctions de la forêt n'est pas l'apanage de la production; elle s'applique aussi en matière de protection par le génie biologique, comme en témoignent les écrits des fondateurs de la politique de restauration des terrains de montagne (RTM), en particulier de Demontzey.

En revanche, la recherche d'un bon état écologique considéré comme une fin en soi n'est guère évoquée jusqu'au début des années 1980. La notion de climax, utilisée dans la formation théorique des forestiers il y a trente ans, reste une image abstraite, avant de tomber dans l'oubli. L'amalgame entre la production optimisée de biens et de services et le substrat naturel de cette production conduira parfois jusqu'à penser et dire, malgré l'histoire, que la forêt ne peut se passer de forestiers.

H.D. : Un forestier non européen décrirait sans doute une histoire assez différente.

Michel Badré : Certainement. En Amérique du Nord, Henry David Thoreau (1817-1862) et John Muir (18381914) s'intéressèrent aux forêts à partir de préoccupations philosophiques ou naturalistes, et réagirent à leur beauté en tant que monuments naturels. Ils ne se souciaient guère des besoins en bois et services d'une société qui, au temps de la conquête de l'Ouest, restait très peu visible dans l'immensité de l'Amérique. Le culte de la wilder$n e s s^{5}$ et la création des parcs nationaux les guidaient, non celui de techniques sylvicoles nouvelles. Et quelques décennies plus tard, Aldo Leopold, dans son Almanach d'un comté des sables ${ }^{6}$, décrit avec un style poétique inimitable ${ }^{7}$ un fonctionnement des forêts du Wisconsin assez proche de ce qu'on réinventera par la suite à propos des écosystèmes terrestres. L'approche explicitement éthique de ces trois auteurs tranche avec celle des auteurs français, pour qui les domaines techniques et éthiques ne relèvent pas

\footnotetext{
${ }^{5}$ Difficilement traduisible, ce terme désigne le caractère sauvage et préservé de la nature idéale. Le courant de pensée plus esthétique que naturaliste associé à ce terme s'attache au respect de la nature préservée, notamment en matière de tourisme ou d'activités sportives. On le trouvera, par exemple, actuellement dans l'association Mountain Wilderness, très active en matière de protection des montagnes du monde.

${ }^{6}$ Publié en 1949, un an après la mort de son auteur, forestier professionnel ayant exercé dans les États de l'Ouest, puis dans le Wisconsin à l'université de Madison.

7 Encore qu' on puisse y trouver une certaine proximité-dans l'humour, le ton pédagogique, la précision technique - avec $\mathrm{La}$ Hulotte, journal «le plus lu dans les terriers", mais dont le renom mérite d'aller bien au-delà.
} 
des mêmes écrits (ce qui ne signifie pas, évidemment, que les techniciens n'ont pas de préoccupations éthiques...)

Plus généralement, deux approches se feront jour dans les pays forestiers importants ou émergents, comme le Chili et la Nouvelle-Zélande, par exemple, l'une centrée sur l'optimisation des usages de la forêt, l'autre, sur la préservation de l'état naturel. Cette dualité entraînera parfois un zonage géographique, surtout à partir de la deuxième moitié du $\mathrm{XX}^{\mathrm{e}}$ siècle, entre forêts dites de production (souvent entièrement issues de plantations artificielles) et forêts naturelles, dans lesquelles les interventions humaines sont quasi inexistantes.

Quant aux pays de la zone intertropicale, en Afrique ou en Amérique, hormis les plantations artificielles récemment introduites, les besoins intenses en bois de chauffage (environ la moitié de la consommation de bois dans le monde porte sur du bois de chauffage), de même que l'écrémage des bois de qualité dans les forêts les plus accessibles, y rendent les notions de gestion durable ou de sylviculture aussi théoriques qu'elles l'étaient en Europe de l'Ouest à la fin du Moyen Âge. Une première étape, indispensable, est ici d'aménager pour réguler les récoltes en fonction des capacités productives locales. Des tentatives allant en ce sens existent, mais elles datent d'à peine quelques décennies et sont loin d'être partout admises... Il y a là, évidemment, un enjeu très fort.

H.D. : Telle que vous décrivez son histoire, la sylviculture présente une étonnante «capacité adaptative», pour reprendre une expression chère aux écologues anglosaxons. Quel bilan peut-on faire de ses succès et, peut être, de ses échecs?

Michel Badré : D'abord, renforçons la modestie traditionnelle $\mathrm{du}$ forestier en notant que ses efforts pour adapter la production à des besoins identifiés n'ont pas toujours été couronnés de succès. Et quand ils l'ont été, c'est souvent pour d'autres raisons que celles des actions forestières mises en œuvre. Ainsi, l'arrêt de la surexploitation des taillis sous futaie en France est due moins aux actions énergiques des forestiers obéissant aux fermes injonctions de Colbert qu'à l'amélioration des rendements agricoles limitant les besoins de défrichement, et au développement de l'usage des combustibles fossiles et de l'électricité. De même, pour la RTM comme pour les plantations du FFN, le délai de quelques décennies entre la décision de mise en œuvre d'une politique et ses premiers effets a entraîné un décalage certain entre les objectifs initiaux et les effets constatés.

Mais une réponse plus concrète appelle à distinguer différents rameaux dans la sylviculture :

- Le premier, très proche de l'agronomie, porte une sylviculture directement attachée à produire une quantité maximale de bois de la qualité optimale, dans les délais les plus courts, en utilisant toutes les ressources de la génétique et des intrants physico-chimiques. Ce rameau est représenté en France par la populiculture, et surtout par le massif du pin maritime aquitain, géré selon ce type de techniques. En d'autres pays, des plantations industrielles - eucalyptus ou résineux à croissance rapide, taillis à courte rotation - ont été largement développées depuis une vingtaine d'années, pour approvisionner les usines de pâte à papier ou de panneaux. Mon sentiment est qu'on ne peut parler de réussite ou d'échec de ces méthodes qu'avec des référentiels d'analyse proches de ceux de l'agriculture : rentabilité économique, maintien de la qualité du patrimoine naturel (notamment des sols, de la biodiversité, etc.), qualité des « services » annexes (paysages, emplois, etc.).

- Le second rameau concilie des objectifs de production avec l'utilisation optimale des processus écologiques. Il correspond à la tendance la plus générale en Europe de l'Ouest, entre des tendances plus ou moins dures, depuis celle du mouvement Prosilva qui fait de l'utilisation des processus naturels une règle quasi intangible, excluant à peu près la régénération artificielle, jusqu'aux orientations sylvicoles plus souplement inspirées du précepte déjà cité, «imiter la nature, hâter son œuvre». On peut penser - ou plutôt : je pense, ayant toujours pratiqué ce type de méthode - que la qualité du patrimoine naturel y est mieux assurée, même s'il s'agit d'un combat permanent : une exploitation mal conduite peut, par exemple, provoquer des tassements de sols irréversibles, même dans une forêt gérée avec le plus grand soin.

- D'autres «rameaux » sont globalement beaucoup plus préoccupants : celui des exploitations des forêts intertropicales, mais aussi de pays en situation économique et politique instable, comme la Russie, et, à moindre degré, la «sylviculture par défaut» que constitue le gain des friches forestières dans les pays développés.

Dans tous les cas, plutôt que de donner arbitrairement des notes de bonne gestion, il me paraît bien préférable de travailler à des outils mondiaux d'appréciation de la qualité de gestion, au regard des critères évoqués plus haut (qui sont ceux de la définition mondiale du développement durable) : maintien de la qualité du patrimoine écologique ; satisfaction des objectifs économiques ; prise en compte des dimensions sociales.

Cette démarche n'a de sens que si elle est mondiale : à défaut, elle crée (ou maintient) des distorsions de compétitivité insupportables entre pays et industries. Des progrès importants, imprévisibles il y a dix ans, ont été faits avec le développement des démarches de certification. Ainsi, le développement des certifications ISO 9001 ou 14001 en forêts publique et privée françaises donne-t-il un outil très fort, adaptable à des référentiels de bonne gestion sur lesquels le débat doit rester ouvert.

Cependant, qui va définir ce qu'est une «bonne gestion »? Cette question masque des enjeux de pouvoir très forts. Et ces enjeux n'ont conduit jusqu'ici qu'à des combats larvés, incompréhensibles pour le public, entre les 
ONG (notamment le WWF, à travers le processus FSC : Forest Stewardship Council) et les acteurs forestiers européens (majoritairement regroupés dans le processus PEFC : Programme for the Endorsement of Forest Certification [Programme de reconnaissance des certifications forestières]). L'intérêt collectif me semble être d'arriver rapidement à un système mondial, fondé sur la reconnaissance mutuelle (techniquement exigeante et crédible, cela va de soi) entre processus divers.

H.D. : Comment, dans ces enjeux, peut-on replacer le mouvement qui conduit à se soucier du bon état écologique de la forêt?

Michel Badré : Ce mouvement dépasse de très loin la forêt. Pour la deuxième fois, après Galilée et Pascal, nous percevons que «le temps du monde fini commence", cette fois parce que nous prenons conscience des effets irréversibles des actions humaines sur le fonctionnement et l'état de la planète. C'est une situation très nouvelle, qui date d'une trentaine d'années. Jusque-là, l'environnement était considéré comme une donnée externe, dont les caractéristiques étaient invariantes, que nos actions ne modifiaient qu'à la marge et localement, et dont les changements influaient sur la vie des hommes sans que l'inverse soit vrai (voir, par exemple, les écrits d'Emmanuel Leroy-Ladurie sur les variations climatiques du dernier millénaire).

Après la grande peur des pluies acides, les accidents climatiques répétés depuis 1980 (tempêtes, dépérissements, sécheresse) ont peu à peu persuadé les forestiers qu'il n'était plus possible de raisonner seulement en termes de «bon usage » de la forêt - pourtant la base même de leur métier, traduite dans un discours de plus en plus élaboré sur la multifonctionnalité. Une réflexion parallèle sur le «bon état » de la forêt s'imposait, pour qui se préoccupait un tant soit peu de la durabilité du «bon usage ». L'émergence de la question des changements climatiques avérés et attendus a renforcé cette interrogation.

Cette émergence d'une attention nouvelle pour le bon état du patrimoine naturel n'est pas propre à la forêt. On la retrouve dans l'inventaire des ZNIEFF - les zones naturelles d'intérêt écologique, faunistique et floristique mené à partir des années 1980 avec une logique d'état et non d'usage, et surtout dans les directives européennes : directive de 1979 sur les oiseaux, de 1992 sur les habitats, centrée sur le «bon état de conservation des habitats », et directive-cadre sur l'eau de 2000, préconisant d'atteindre en 2015 un «bon état écologique » des eaux terrestres. Que le bon état écologique ainsi visé ne soit pas défini - ce qui est sans doute sage - n'enlève rien à la signification d'une démarche très nouvelle par rapport à celles de l'homme domestiquant, avec plus ou moins de bonheur, une nature supposée bien portante.

H.D. : Existe-t-il cependant une spécificité forestière en matière de gestion des ressources naturelles?
Michel Badré : Je crois que la spécificité forestière en la matière est double : par ses échelles de temps, la forêt représente un marqueur à effets retardés mais durables, et par son caractère emblématique, une image de la nature préservée dans un monde anthropisé.

Pour la société, les peurs liées à une dégradation de la forêt sont fortes (à juste titre, sans doute, dira le forestier. . .). L'impact environnemental de l'agriculture reste une question de spécialistes touchant peu le grand public $^{8}$, et les débats sur la protection d'espèces emblématiques telles que le loup et l'ours relèvent souvent de la polémique. Au contraire, la dégradation des forêts a un impact fort sur la société : il s'agit de monuments naturels, au même titre que la haute montagne, les déserts ou les océans.

Le dispositif d'observation des forêts d'Europe, mis en place dans les années 1980, tente de répondre à cette inquiétude. Et la démarche est riche d'enseignements, à trois niveaux d'analyse :

- les réseaux statistiques de suivi des effets de la pollution et de l'état sanitaire (réseau à mailles carrées $16 \times 16 \mathrm{~km}$, pour l'essentiel) ;

- les réseaux de suivi de l'évolution des écosystèmes forestiers (en France, 100 placettes du réseau RENECOFOR, en Europe 500 à 600 placettes comparables;

- les sites-ateliers lourdement instrumentés, essentiellement pour étudier les cycles du carbone et des éléments minéraux.

Ce dispositif scientifique a déjà fourni beaucoup d'éléments de compréhension sur l'état écologique des écosystèmes forestiers. Mais son intérêt principal réside dans sa pérennité.

H.D. : On voit en effet l'intérêt d'assurer une telle pérennité, précisément en relation avec le changement climatique global. Quelles réactions ce changement suscite$\mathrm{t}$-il chez les forestiers?

Michel Badré : C'est d'abord en matière de résistance et de résilience face aux tempêtes que les forestiers, instruits pas les accidents climatiques à répétition des années 1980 et 1990, ont cherché à réagir. L'intensification des prélèvements en éclaircie, l'accent mis sur les mélanges d'essences et de structures de peuplement, l'évolution des techniques de reconstitution relèvent de ces démarches.

Il reste beaucoup à faire en matière d'adaptation des peuplements aux extrêmes climatiques, et en particulier à la sécheresse. Les travaux menés depuis quelques années en écophysiologie semblent montrer de façon nette

\footnotetext{
8 Sauf lorsqu'elle pose des questions de sécurité sanitaire ou alimentaire : cette situation évolue donc assez vite, comme l'indique la montée des préoccupations sur les pesticides ou les nitrates, par exemple.
} 
l'intérêt de baisser l'indice foliaire ${ }^{9}$ des peuplements pour les rendre plus résistants à la sécheresse : l'utilisation de ce type de données dans la conduite des peuplements devrait se répandre rapidement.

D'autres thèmes font encore l'objet de débats, l'intuition semblant y précéder la validation scientifique, notamment en matière d'effet du traitement régulier ou irrégulier sur la stabilité des peuplements. Et l'évolution actuelle et probable des aires de répartition des principales espèces forestières fait maintenant l'objet de travaux concordants. On peut s'attendre, en particulier, à une régression assez forte de l'aire de répartition optimale du hêtre, essence la plus sensible à une bonne alimentation en eau.

Voilà en tout cas des éléments que la gestion doit évidemment intégrer dès maintenant. . .

H.D. : À ce propos, on peut imaginer l'existence de débats internes relayant ceux de la société, actuellement comme dans le passé. Comment la société dans son ensemble intervient-elle dans ces débats?

Michel Badré : Malgré son apparent isolement, la collectivité forestière a le plus souvent reflété, dans ses débats internes, les courants de pensée qui parcouraient la société à la même époque. La pensée de Duhamel du Monceau est celle de Buffon et des physiocrates; la foresterie du $\mathrm{XIX}^{\mathrm{e}}$ siècle, dans sa recherche de clarifications mathématiques ${ }^{10}$, me semble assez marquée par les tendances scientistes de l'époque; le FFN est un reflet forestier de la période des trente glorieuses; et on pourra rechercher dans les courants forestiers actuels toutes les tendances allant de l'écologie fondamentale au productivisme le plus poussé.

Mais la société a pesé sur les débats forestiers surtout par ses évolutions structurelles profondes, et non par ses mouvements d'idée, encore moins, on l'a vu, par ses demandes explicites... La démographie, les besoins en terres pour la culture et l'élevage, puis l'augmentation des rendements agricoles et l'exode rural ont été les déterminants les plus forts de la variation des surfaces forestières ${ }^{11}$ et de l'intensité des exploitations en France, bien avant les politiques volontaristes ou l'action des forestiers. Cultivant à nouveau la modestie des forestiers, on pourrait dire que la France s'est déboisée malgré leurs efforts (y compris ceux de Colbert : la diminution

\footnotetext{
${ }^{9}$ Rapport de la surface totale des feuilles à la surface projetée au sol du houppier.

10 De la méthode d'aménagement du «quartier bleu » à celle du contrôle, ou aux calculs de taux de rentabilité économique de Faustmann.

11 On rappellera que la surface forestière française, probablement de l'ordre de 40 Mha au temps de la «Gaule chevelue » de César, est passée à environ 7 Mha en 1800, puis à environ 11 Mha (Alsace et Moselle incluses) au temps de l'inventaire Daubrée (1912), et à plus de 16 Mha actuellement, avec une progression régulière $\mathrm{d}^{\prime}$ environ $+0,5 \%$ par an. .
}

des surfaces forestières et la surexploitation se sont poursuivies 150 ans après lui...), et qu'elle se reboise bien au-delà de ce que peut expliquer leur action.

S'ajoutent maintenant à ces tendances la préoccupation générale sur l'état de la planète, et le besoin de débat et de transparence qui caractérise une société de communication. Cela obligera de plus en plus à soumettre au débat public les orientations de politique forestière, à tous les niveaux, du plus local au plus global. Cette démarche n'est pas encore naturelle aux forestiers, qui y voient parfois une intrusion du grand public (par définition moins qualifié qu'eux au plan technique) dans leur sphère d'action. Refuser ce débat serait pourtant, à mon avis, une erreur stratégique lourde, alors que la légitimité des forestiers ne tient, on l'a vu, qu'à leur aptitude à répondre aux attentes de la société : la difficulté réelle est pour eux d'éclairer les débats, notamment sur la compatibilité des choix de préservation et de développement. Des débats qu'il s'agit de ne pas occulter ni de s'approprier exclusivement.

Là encore, le débat sur les pluies acides est plein d'enseignements : parti du grand public et non de la communauté forestière, il a suscité chez elle un certain agacement. Largement repris (je n'ose pas dire : récupéré... ) par la communauté scientifique et forestière, et par les instances politiques européennes, il a permis de déboucher sur des outils de suivi et de diagnostic dont plus personne ne conteste aujourd'hui l'intérêt. Comme quoi des questions mal posées peuvent parfois conduire à de bonnes réponses...

H.D. : Ceci nous amène à la question des relations entre la recherche et la gestion des forêts. Vous y avez beaucoup réfléchi : comment avez-vous évolué sur ce sujet, notamment à la lumière de vos activités à l'Office national des forêts, puis au groupement d'intérêt public Écofor?

Michel Badré : Le praticien de la gestion, nonchercheur, que je suis perçoit en effet un changement très profond dans la relation entre la recherche et l'application de ses résultats. Il y a trente ans, j'avais retenu que le précepte « mieux connaître et mieux comprendre, pour mieux gérer » définissait le fonctionnement idéal du couple chercheur-gestionnaire. Le gestionnaire posait des questions (à défaut, le chercheur les imaginait luimême), le chercheur observait, expérimentait, déduisait, fournissait des résultats, et ceux-ci étaient ensuite transférés au gestionnaire par des mécanismes ou des structures de développement. Les incompréhensions étaient certes nombreuses - questions mal posées ou jugées sans intérêt, réponses pas claires, développement inefficace, etc. -, mais chacun y trouvait pourtant à peu près son compte.

Ce système, d'inspiration très cartésienne, a obtenu des résultats tout à fait remarquables : on ne citera que l'exemple, peut-être le plus significatif, de la génétique forestière, qui s'est construite selon ce schéma depuis 
une cinquantaine d'années, avec des résultats largement utilisés dans le monde de la gestion.

Au début des années 1980, les «pluies acides » ont porté un coup assez sévère à ce schéma : des questions issues du grand public, faisant référence avec de fortes inquiétudes à la problématique des changements globaux qui était née peu avant dans la communauté scientifique, ne permettaient pas de mener dans les délais attendus des démarches de recherche abouties. Cela conduisit à des réponses en termes d'outils ou de structure de suivi et de recherche, et non en termes de résultats : réseaux systématiques d'observation, sites-ateliers, création du groupement d'intérêt public Écofor pour coordonner les recherches à mener sur ces questions, à partir des premiers résultats de mesures.

Le succès d'une telle démarche suppose une liaison d'un nouveau type entre recherche et gestion, le transfert des questions et des réponses entre l'une et l'autre ne relevant plus de la même logique linéaire, mais nécessitant beaucoup plus d'interactivité.

H.D. : À quelles conditions peut se développer ce nouveau type de liaison?

Michel Badré : Les phénomènes objets de recherche (ici, les changements climatiques, ou les cycles biogéochimiques) obéissent maintenant à des pas de temps du même ordre que les cycles de production forestière et que les délais de production de résultats, ce qui oblige à agir en situation d'incertitude et non dans un cadre éclairé par des recherches abouties.

Les tempêtes de 1999, puis la sécheresse de 2003 ont habitué tous les acteurs forestiers à ce mécanisme d'interaction entre constats d'expérience, processus de recherche et décision de gestion. Les « expertises collectives " menées à ces deux occasions ont montré à la fois l'utilité et la difficulté de ces exercices - peut-être par défaut d'un réel métier d'expertise capable d'assurer l'interface entre recherche et gestion, alors que les disciplines en cause sont très diverses et entrecroisées. L'interface recherche/gestion ne se résume plus à un transfert par le biais du développement : elle suppose maintenant de l'expertise, ce qui est autre chose. Et développer ce chaînon manquant apparaît comme la condition de l'exercice réel des principes de précaution et de responsabilité, par le politique comme par le gestionnaire, face aux questions scientifiques très complexes posées par les changements globaux.

La dialectique entre bon usage et bon état, que j'ai évoquée plus haut, doit aussi beaucoup à la communauté scientifique. Si le bon état écologique n'est pas défini dans les directives de nature politique, les chercheurs estiment, à juste titre, qu'il leur appartient de s'en préoccuper. C'est ainsi que les travaux sur l'augmentation de la productivité des forêts se sont accompagnés de programmes de recherche lourds sur les cycles biogéochimiques : ces programmes sont au cœur des travaux sur l'impact des changements globaux menés dans les sites-ateliers de l'Observatoire de recherche en environnement sur la forêt.

Mais ce changement d'approche de l'articulation recherche/gestion cache une évolution plus philosophique. Le phénomène des changements globaux, dans toute sa complexité, oblige à une action collective face à une situation qui concerne, pour la première fois peut-être, l'humanité entière. Il oblige aussi à passer d'une approche «prométhéo-cartésienne », où on transforme le monde après l'avoir analysé et compris, à une autre, plus humble mais non moins active, où l'action se définit chaque jour dans un monde changeant, en interaction permanente avec la recherche et l'observation de la vie.

H.D. : De ce point de vue, les tempêtes de décembre 1999 vous paraissent-elles avoir livré tous leurs enseignements?

Michel Badré : Oui, je crois, pour l'essentiel, dans le domaine des « biotechniques ». L'expertise collective menée depuis cinq ans sous la conduite du GIP Écofor, et en particulier d'Yves Birot, a été très riche d'enseignements dans ce domaine.

Non, toujours à mon avis, dans le domaine économique, où les propositions de recherche émanant de la communauté scientifique sont restées peu nombreuses. Ainsi, nous ne disposons d'aucune analyse rétrospective de l'efficacité du système d'aides publiques mis en place, alors que la pertinence et le calibrage de chaque type d'aide, en situation d'urgence, posent des questions très complexes. De même, et j'en parle en connaissance de cause pour avoir participé dans l'urgence à son élaboration collective dans les quinze premiers jours de janvier 2000 , nous n'avons pas d'analyse scientifique du système mis en place pour commercialiser les chablis, système très différent des pratiques habituelles.

Non, à nouveau, dans le domaine de la gestion de crise. Là aussi, de nombreuses décisions, bonnes ou mauvaises, ont dû être prises à la hâte. Les diverses missions administratives menées, ou l'élaboration de "guides de crise » à partir du retour d'expériences, ne remplacent pas une analyse scientifique objective, faite avec le recul suffisant. À défaut, la place était largement ouverte aux jugements hâtifs et aux polémiques, désagréables pour les acteurs directement concernés, et qui ne seront pas d'un grand secours aux gestionnaires de la prochaine crise semblable... Mais sans doute est-il encore temps de s'y attaquer.

H.D. : Revenons aux forêts tropicales. Les chercheurs français ont largement contribué à leur connaissance et, en dépit d'énormes difficultés, y poursuivent un travail mondialement respecté, par exemple en Guyane, mais aussi en divers pays d'Afrique, d'Asie et d'Océanie. Et vous parliez tout à l'heure d'un enjeu très fort. . .

Michel Badré : J'évoquerai cette question, là aussi, en non-spécialiste, mon activité professionnelle ayant 
toujours porté principalement sur les forêts de la zone tempérée.

La Guyane présente, selon les spécialistes de la biodiversité, une richesse exceptionnelle (comme les autres forêts naturelles de la zone tropicale humide). Le rôle de la France est manifestement de préserver cette richesse et de développer les recherches sur ces milieux. La compréhension du fonctionnement d'écosystèmes aussi complexes me semble être d'un grand intérêt pour toute la science forestière, en dehors même de l'enjeu mondial que représente la lutte contre la perte de biodiversité. Elle est aussi à la base d'une gestion raisonnée de ces écosystèmes, y compris pour une récolte ligneuse raisonnée.

Mais le fonctionnement d'écosystèmes artificiels en zone tropicale - plantations industrielles sur lesquelles la France travaille au Congo notamment - justifie aussi des actions lourdes : le monde développé peut en effet difficilement refuser aux pays du tiers-monde le droit à une valorisation économique de leur patrimoine, dans un seul but de protection de la biodiversité mondiale. La demande forte, exprimée par les pays concernés, d'un transfert de capacités de recherche me paraît au demeurant tout à fait légitime, à partir de ce qui est fait par la communauté scientifique française.

La problématique du stockage du carbone dans ces forêts justifie aussi un travail de recherche approfondi : même si, comme par le passé, les futures négociations internationales sur le sujet se concluent par des arbitrages plus politiques que scientifiques, il est important d'asseoir ces négociations sur des bases scientifiques aussi solides que possible.

J'ajouterai que, sur l'ensemble des questions relatives à la préservation des forêts tropicales, un gros effort de clarification des enjeux est indispensable. Compliqué par des difficultés géopolitiques évidentes, cet effort me semble pourtant indispensable pour aboutir à des décisions de préservation pertinentes.

H.D. : Et en dehors des forêts tropicales?

Michel Badré : Bien qu'on en parle beaucoup moins, je souhaiterais évoquer aussi le cas des forêts russes, et surtout bien sûr du massif sibérien. Leur gestion durable ne me semble pas faire l'objet actuellement de beaucoup d'attention de la part de la communauté mondiale, étrangement silencieuse ici, alors que les enjeux écologiques et économiques m'en paraissent importants : ce sujet justifierait par exemple plus d'efforts en matière de certification de la gestion durable que les rivalités locales entre tenants des différents processus dans les forêts européennes, qui me semblent objectivement beaucoup moins menacées.

H.D. : Votre activité actuelle à l'Inspection générale de l'environnement vous conduit à aborder des questions environnementales nouvelles, parfois éloignées de celles du domaine forestier. Que vous apporte votre expérience forestière?
Michel Badré : D'abord, un certain étonnement : alors que les questions apparaissent très semblables, en matière de connaissance, de suivi et de gestion de milieux, de recherche ou d'articulation entre écologie et économie, j'ai parfois l'impression de deux mondes qui s'ignorent (je relèverai simplement ici que j'ai à peu près totalement renouvelé mon carnet $\mathrm{d}^{\prime}$ 'adresses professionnelles depuis deux ans...). L'absence de culture du management (au sens de structuration des processus de décision et de gestion collective) du monde de l'environnement ne peut aussi que surprendre, pour qui vient du monde de la forêt.

Ensuite, un peu de frustration de voir peu utilisée, dans le domaine des milieux naturels non forestiers, l'expérience d'organisation de la gestion de milieux, développée depuis longtemps dans le monde forestier. En particulier, la notion de plan de gestion, qui s'est maintenant implantée partout en matière de milieux naturels, aurait sans doute beaucoup à apprendre (y compris dans les erreurs à ne pas refaire) de l'expérience des aménagements forestiers, outils de programmation des actes de gestion à partir des connaissances scientifiques et techniques et des objectifs retenus pour le territoire.

À l'inverse, l'ouverture du monde de l'environnement sur la société (principalement, mais pas uniquement) par le biais du monde associatif est pleine d'enseignements pour un forestier.

Enfin, un point commun dominant : pour sortir du discours parfois désespérément confus et flou sur le développement durable, il faut savoir mettre en regard des projets ou des activités économiques, des enjeux de bon état écologique et la nature de la demande sociale. . . et savoir les hiérarchiser ou les intégrer. Cette question est posée aussi bien par l'avenir du Marais poitevin entre agriculture de production et préservation d'une zone humide exceptionnelle, par celui de la basse Seine entre développement des ports et fonctionnement écologique de l'estuaire, ou, d'une façon générale, par l'application de l'article 6 de la directive européenne de 1992 sur les habitats, en matière d'impact de projets nouveaux sur les sites du réseau Natura 2000. Cette question est aussi celle que rencontre tout gestionnaire forestier de l'échelle la plus locale à celle des forêts du monde. Et la réponse demande des compétences techniques, spécialisées et transversales qu'il est indispensable de développer, dans le domaine de la recherche comme dans celui de la gestion des milieux naturels.

H.D. : Ce qui nous ramène à la question des moyens financiers...

Michel Badré : En effet, au-delà des compétences humaines, et hors tout propos démagogique, il faut rappeler qu'une politique de préservation d'un bien commun tel que la biodiversité dans les espaces naturels, forestiers ou non, nécessite des moyens financiers collectifs. Les discours entendus, dans le monde forestier comme dans 
celui de l'environnement, me paraissent ici souvent trop réducteurs. Il n'est plus possible à certains forestiers de parler de financement des "contraintes environnementales », à côté du discours convenu sur la gestion durable, comme si tout ce qui n'est pas lié à la production était une contrainte. Il n'est plus possible non plus à certains représentants du monde de l'environnement (ou des finances...) de laisser penser que la gestion des documents d'objectif Natura 2000 n'aura qu'un coût très faible, et qu'elle peut donc être menée à l'amiable, de proche en proche, en internalisant dans le budget des producteurs agricoles et forestiers l'essentiel des dépenses nouvelles de préservation.

À titre indicatif, l'estimation actuelle des mesures nouvelles de mise en œuvre des documents d'objectif Natura 2000, faite par la direction de la Nature et des Paysages du ministère de l'Écologie et du Développement durable, est de l'ordre de $300 \mathrm{M} €$ par an, soit le triple du budget consacré par ce ministère à sa politique de préservation des espèces et des espaces sensibles : cela ne peut se gérer par voie d'expédients ou de redéploiements à la marge. Il est normal d'appliquer un principe directement dérivé du principe «pollueur payeur » à celui dont l'activité - agricole, forestière ou autre - dégrade le patrimoine, en contravention avec des normes réglementaires (qu'il faut d'ailleurs peut-être revoir). Mais il serait illusoire de penser que des dépenses nouvelles importantes, sans retour sur investissement pour le propriétaire ou le gestionnaire, vont être prises en compte par la seule bonne volonté des acteurs économiques individuels, par voie amiable, sans financement à hauteur des enjeux. À défaut d'une approche réaliste de cette question, on peut en tout cas s'attendre à une floraison rapide de contentieux européens à impact financier très élevé.

H.D. : Une approche réaliste : n'est-ce pas, au fond, ce qui pourrait caractériser votre propos?

Michel Badré : Une approche enracinée dans le temps présent, surtout : on croit souvent (et j'ai cru, à mes débuts) que le forestier a la chance de vivre hors du temps, ou en tout cas dans des échelles de temps qui échappent au commun des mortels. Cela lui permet un grand détachement par rapport aux contingences immédiates.

Je crois maintenant que c'est une idée fausse, et assez irresponsable. On a vu que les pas de temps des changements globaux, en se contractant, étaient devenus plus courts que ceux de la production forestière. Mais, dans bien d'autres domaines, la plupart des actions lourdes auxquelles j'ai participé, dans ma vie professionnelle de forestier, étaient marquées par des échéances courtes, dans le temps long de la forêt : améliorer l'emploi des ouvriers, réagir aux conséquences d'une tempête, implanter le réseau Natura 2000, chercher des perspectives nouvelles pour un établissement de 11000 personnes en pleine crise, concilier performances et solidarités dans une communauté de travail allant des ouvriers forestiers aux cadres dirigeants, tout cela relève du court terme autant que du long.

Et cela nécessite, dès l'instant présent, les mêmes moyens qu'ailleurs : ce n'est pas parce que la gestion est durable qu'elle ne doit pas commencer tout de suite...

To access this journal online: www.edpsciences.org 